Pacific Journal of Mathematics

ON MEASURABILITY OF STOCHASTIC PROCESSES IN
PRODUCTS SPACE 


\section{ON MEASURABILITY OF STOCHASTIC PROCESSES IN PRODUCTS SPACE}

\section{JACOB FeLdMAN}

1. Introduction. Let $\mathscr{E}$ be a $\sigma$-algebra of subsets of $X$, and $T$ a set. Let $\Omega=X^{T}$, and let $\mathscr{C}$ be the $\sigma$-algebra of subsets of $\Omega$ generated by the finite cylinder sets, i.e., sets of the form $\Lambda=\left\{\omega \in \Omega \mid \omega\left(t_{1}\right) \in A_{1}, \cdots\right.$, $\left.\omega\left(t_{n}\right) \in A_{n}\right\}, A_{1}, \cdots, A_{n} \in \mathscr{X}$. Let $P_{0}$ be a probability measure on $\mathscr{C}$. Thus the coordinate variables $x_{t}(\omega)=\omega(t), t \in T$, are the Kolmogorov version [5] of the stochastic process with joint distributions $F_{t_{1}}, \cdots,{ }_{t_{1}}\left(A_{1}, \cdots\right.$, $\left.A_{n}\right)=P_{0}\{\Lambda\}$. For various purposes, it is appropriate to enlarge this $\sigma$ algebra and extend the measure. In the present paper two methods of doing this will be mentioned, and one of the methods will be studied.

[A] Suppose $X$ is a compact Hausdorff space and the Borel sets. Then $\Omega$ is a compact Hausdorff space in the product topology. A straightforward application of the Stone-Weierstrass theorem and the Riesz-Markov theorem shows that there is a unique regular measure on the Borel subsets $\mathscr{S}$ of $\Omega$ which agrees with $P_{0}$ on $\mathscr{C}$, provided the finite-dimensional marginal measures are all regular. We call this measure $P$. This idea is due to S. Kakutani [3], and was discussed in detail by E. Nelson [8].

[B] By a condition is meant a set-valued function $k$ from $T$ to $\mathscr{X}$. For any condition $k$, we define

$$
\begin{aligned}
\Gamma(k) & =\{\omega \mid \omega(t) \in k(t) \text { for all } t \in T\}, \text { and } \\
\Gamma(S, k) & =\{\omega \mid \omega(t) \in k(t) \text { for all } t \in S\},
\end{aligned}
$$

$S$ being a subset of $T$. It is possible to extend $P_{0}$ to a class of sets of the form $\Gamma(k)$, as follows.

The following lemma is a straightforward generalization of the separability lemma in [1], p. 56.

Lemma 1.1. For any condition $k \exists$ a countable set $S \subset T$ such that $P_{0}\{\Gamma(S, k)-\Gamma(\{t\}, k)\}=0$ for all $t \in T$.

The proof is a simple exhaustion argument. Such a countable subset $S$ will be called determining for $k$.

Let $\mathscr{K}$ be a family of sets with the properties

(i) $X \in \mathscr{K}$

Received March 27, 1961. Written partly with support from Contract NONR 222 (60) and partly while an NSF fellow. 
(ii) any countable subfamily of $\mathscr{K}$ with the finite intersection property (F.I.P.) has nonnull intersection. Such a family will be called countably compact. If (ii) holds without the countability restriction, then $\mathscr{K}$ is called compact. If a condition $k$ has values in $\mathscr{K}$, it will be called a $\mathscr{K}$-condition.

The set of positive integers will be written $I$. Unions and intersections whose index set is $I$ will be written simply $\bigcup_{j}$, etc. rather than $\bigcup_{j \in I}$, etc. The following result can then be proven. It is stated in [7].

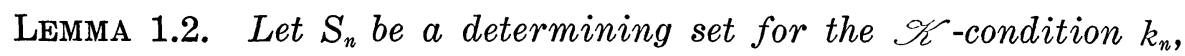
$n \in I$. Let $\Delta=\mathrm{U}_{n}\left\{\Gamma\left(S_{n}, k_{n}\right)-\Gamma\left(k_{n}\right)\right\}$. Then $\Delta$ has inner $P_{0}-$ measure 0 .

$\mathscr{C}_{\mathscr{F}}$ is now defined to be those subsets $\Gamma$ of $\Omega$ such that $\exists \Gamma^{\prime}$ in $\mathscr{C}$ with $\left(\Gamma-\Gamma^{1}\right) \cup\left(I^{1}-\Gamma\right)$ subset of a set of the form of $\Delta$ in the above lemma. These sets $\Gamma$ form a $\sigma$-algebra, and the assignment to $\Gamma$ of the same measure as the $P_{0}$-measure of $\Gamma^{1}$ determines unambiguously a measure $P_{\mathscr{K}}$ on $\mathscr{C}_{\mathcal{Z}}$, which is an extension of $P_{0}$. This construction, based on ideas of Doob and Khintchine [4] is done by A. Mayer in [6], [7].

REMARK 1.1. Notice that $\mathscr{C}_{\mathscr{K}}$ contains all sets of the form $\Gamma(k)$, for any $\mathscr{K}$-condition $k$, assigning to such a set the measure $P_{0}\{\Gamma(S, k)\}$, $S$ being any determining set for $k$.

Remark 1.2. If $X$ is compact Hausdorff, $\mathscr{X}$ the Borel sets, $\mathscr{K}$ the compact sets, and $P_{0}$ satisfies the regularity condition of $[A]$, then $\mathscr{C}_{\mathscr{Z}} \subset \mathscr{B}$, and $P \mid \mathscr{C}_{\mathscr{K}}=P_{\mathscr{K}}$. This is a consequence of the following (under the hypotheses of the last sentence):

LEMMA 1.3. If $S$ is determining for the condition $k$, and $k(t)$ is compact for all $t$, then $P\{\Gamma(k)\}=P\{\Gamma(S, k)\}$.

Proof. By Theorem 2.2 of [3] there is some countable subset $S_{1}$ of $T$ such that $P\left\{\Gamma\left(S_{1}, k\right)\right\}=P\{\Gamma(k)\}$. Now, $\Gamma\left(S_{1}, k\right) \supset \Gamma\left(S \cup S_{1}, k\right) \supset \Gamma(k)$, so $P\left\{l^{\prime}\left(S \cup S_{1}, k\right)\right\}=P\left\{I^{\prime}(k)\right\}$. But

$$
\Gamma(S, k)=\Gamma\left(S \cup S_{1}, k\right) \cap \bigcap_{s \in S}\left\{\Gamma\left(S_{1}, k\right)=\Gamma(\{s\}, k)\right\} .
$$

Thus $P\{\Gamma(S, k)\}=P\left\{\Gamma\left(S \cup S_{1}, k\right)\right\}$.

We will deal mainly with the situation where $T$ is a topolozical space, and with a certain $\sigma$-subalgebra $\mathscr{D}_{\mathscr{K}}$ of $\mathscr{C}_{\mathscr{L}}$, where $\mathscr{D}_{\mathscr{K}}$ is defined like $\mathscr{C} \mathscr{K}$, except that the only conditions $k$ usad for $\mathscr{D} \mathscr{\mathscr { C }}$ will be those of the form 


$$
\begin{aligned}
k(t)= & K \text { for } t \in U \\
& X \text { for } t \notin U,
\end{aligned}
$$

$U$ being an open set in $T$, and $K \in \mathscr{C}$. For such a $k$, we write $\Gamma(k)$ as $\angle(U, K)$. The restriction of $P_{\mathscr{K}}$ to $\mathscr{D} \mathscr{K}$ will be called $Q_{\mathscr{K}}$.

If $\mathscr{K}$ consists of closed sets in a metric space, $T$ is locally compact, and $\tau$ is a regular measure on $T$, then $\left(\mathscr{D}_{\mathscr{K}}, Q_{\mathscr{K}}\right)$ has the convenient property that whenever the map $t \rightarrow x_{t}$ (where $x_{t}(\omega)=\omega(t)$ ) is measurable in probability, i.e. is continuous in probability outside of some $\tau$-null set, then the map $(\omega, t) \rightarrow \omega(t)$ can be made measurable the $\mu \times \tau$-completion of $\mathscr{A} \times \mathscr{T}$, where $\mathscr{T}$ is the Borel sets of $T$ and $(\mathscr{K}, \mu)$ some extension of $\left(\mathscr{D}, \mathscr{K} Q_{\mathscr{K}}\right)$. (See [7], Theorem 2.) This says, in a sense, that $\mathscr{D}_{\mathscr{F}}$ is " not too large." On the other hand, it is "not too small," in the sense that it contains many natural subsets which are not in ' $\mathscr{C}$; this will be shown.

In $\S 2$ are given some examples and general remarks concerning compact and countably compact families.

In [8], with $X$ and $T$ compact metrizable spaces, various natural subsets of $\Omega$ and $\Omega \times T$ were shown to be in $\mathscr{B}, \overline{\mathscr{S}}$, or product $\sigma$ algebras derived from them (the bar over a $\sigma$-algebra signifies completion with respect to the measure being considered on it). In $\S 3$ and 4 we show (in a somewhat more general context) that these subsets are in $\mathscr{D}_{\mathscr{K}}, \overline{\mathscr{D}}_{\mathscr{K}}$, or the corresponding product $\sigma$-algebrans, where $\mathscr{K}$ is a countably compact family of closed subsets of $X$ which contains a complete system of neighborhoods for each point of $X$ (or, briefly, generates the topology of $X$ ).

\section{Some topological considerations.}

Lemma 2.1. Let $X$ be a 1-st countable Hausdorff space. Then any countable compact family $\mathscr{C}$ of subsets of $X$ which generates the topology of $X$ consists of closed sets only.

Proof. Suppose $K \in \mathscr{K}$, and $x \notin K$. Choose a countable family $\left\{K_{n} \mid n \in I\right\}$ of neighborhoods of $x$ in $\mathscr{C}$, with $\bigcap_{n} K_{n}=\{x\}$. If $x \in \bar{K}$, then $K \cap K_{1} \cap \cdots \cap K_{n}$ is never empty. Thus, $K \cap \cap_{n} K_{n}$ is nonempty, so $x \in K$.

REMARK 2.1. If we assume that $X$ actually has a countable base for its open sets, then clearly any intersection of sets of $\mathscr{K}$ can be reduced to a countable intersection. In particular, it follows that . $\mathcal{K}^{\prime}$ is actually a compact family, not just countably compact.

LEMma 2.2. (Alexander). Let $\mathscr{K}$ be a compact family of subsets 
of a set $X$. Let $\tilde{\mathscr{K}}$ be the family of arbitrary intersections of finite unions of sets of then $\tilde{\mathscr{K}}$ is closed under arbitrary intersections and finite unions, and is again a compact family.

\section{Proof. See [9], p. 139.}

COROLlaRY 2.1. The most general compact family of sets on a set $X$ arises by choosing a subfamily of the closed sets, for some compact topology on $X$.

Proof. Given a compact family $\mathscr{K}$ on a set $X$, use $\tilde{\mathscr{K}}$ as the family of closed sets for $X$; this gives a compact space.

REMARK 2.2. The property of countable compactness does not persist from $\mathscr{K}$ to $\tilde{\mathscr{K}}$. For example, let $A$ be all ordinals up to and including the first uncountable ordinal $\alpha_{0}$. Let $B$ be the rational numbers $\left\{0 ; 1, \frac{1}{2}, 1 / 3, \cdots\right\}$. Let $X=A \times B-\left\{\left(\alpha_{0}, 0\right)\right\}$. Let $\mathscr{\varkappa}^{\prime}$ consist of all sets of the form $K_{\alpha, n}=\left\{\left(\alpha^{1}, x\right) \mid \alpha^{1}>\alpha, x<1 / n\right\}$, where $\alpha$ is a countable ordinal and $n \in I$. Then no countable intersection of sets $K_{\alpha, n}$ is empty, so $\mathscr{K}$ is countably compact. But let $L_{n}=\bigcap_{\alpha<\alpha 0} K_{\alpha, n}=\left\{\left(\alpha_{0}, x\right) \mid x<1 / n\right\}$. Then the $L_{n}$ have the F.I.P., but $\bigcap_{n} L_{n}=\phi$.

In $\S 3$ we shall be considering countably compact families $\mathscr{K}$ on separable metrizable spaces $X, \mathscr{K}$ generating the topology of $X$. Some examples follow.

(a) $X$ a Banach space which is separable and a dual, $\mathscr{Y}$ the set of all closed spheres. This is mentioned in [6].

In this connection, however, notice that the separable Banach space $\mathbf{C}$ of all continuous functions on, say, the closed interval $[-1,1]$, is not a dual; and, in fact, the family of all closed spheres in this Banach space is not a countably compact family. To see this, let

$$
f_{n}(\lambda)=\left\{\begin{array}{l}
1 \text { if }-1 \leqq \lambda \leqq 0 \\
1-n \lambda \text { if } 0<\lambda<\frac{1}{n} \\
0 \text { if } \frac{1}{n} \leqq \lambda \leqq 1,
\end{array}\right.
$$

and let $f_{n}^{\prime}(\lambda)=-f_{n}(-\lambda)$. Let $K_{n}$ be the closed sphere of radius 2 about $f_{n}-2$, and $K_{n}^{\prime}$ the closed sphere of radius 2 about $f_{n}^{\prime}+2$. Then

$$
K_{n} \cap K_{n}^{\prime}=\left\{g \mid f_{n}^{\prime} \leqq g \leqq f_{n}\right\} \neq \phi .
$$

Since $f_{1} \geqq f_{2} \geqq \cdots$ and $f_{1}^{\prime} \leqq f_{2}^{\prime} \leqq \cdots$, we have $K_{1} \cap K_{1}^{\prime} \supset K_{2} \cap K_{2}^{\prime} \supset \cdots$. Thus, the spheres $\left\{K_{n}, K_{n}^{\prime} \mid n=1,2, \cdots\right\}$ have the F.I.P., but there is 
no continuous function in their intersection. The author does not know, however, whether some $\mathscr{L}^{\sim}$ does not exist for $\mathrm{C}$.

(b) An example where the metric space is not complete: let $X$ be the nondyadic numbers in the unit interval. $\%$ will be defined as follows. Let $S_{n}$ be the set of dyadics of the form $k / 2^{n}, k=0, \cdots, 2^{n}$. Then $X=[0,1]-\bigcup_{n} S_{n}$. Let $\mathscr{S}_{n}$ be the intersection with $X$ of intervals [a, b], where $a=(k+1 / 8) 1 / 2^{n}, b=(k+7 / 8) 1 / 2^{n}, k=0,1, \cdots, 2^{n}-1$. Let $\mathscr{K}=\bigcup_{n} \mathscr{K}_{n}$.

To see that $\mathscr{r}$ generates the topology of $X$, we must show that any $x \in X$ is an interior point of some interval in $\mathscr{K}_{n}$, for arbitrarily large $n$. But a nondyadic number $x$ is characterized by the property that a zero followed by a one occurs arbitrarily far out in its dyadic expansion. Thus, for arbitrarily large $n$, we can get $k / 2^{n}+1 / 2^{n+2}<x<k / 2^{n}+1 / 2^{n+1}$, so that $x$ is interior to an interval of $\mathscr{K}_{n}$.

To see that $\mathscr{K}^{-}$is countably compact, suppose we have a sequence $K_{1}, K_{2}, \cdots$ with the F.I.P. Assume repetitions have been eliminated. Then no two can come from the same $\mathscr{K}_{n}$, since two members of $\mathscr{K}_{n}$ are either identical or disjoint. Consider now the closed intervals $\bar{K}_{n}$ in $[0,1]$. These have the F.I.P., and are closed in $[0,1]$. Thus their intersection is nonempty. Further, let $K_{n} \in \mathscr{K}_{i_{n}}$. Then $\bar{K}_{n} \cap S_{i_{n}}=\phi$, so $\left(\bigcap_{n} \bar{K}_{n}\right) \cap\left(\bigcup_{m} S_{i_{m}}\right)=\phi$. Since $i_{m}$ does not repeat itself, and since $S_{1} \subset S_{2} \subset \cdots$, we have $\bigcup_{m} S_{i_{m}}=\bigcup_{n} S_{n}$. Thus, $\left(\bigcap_{n} \bar{K}_{n}\right) \cap X \neq \phi$. But this is the same as $\bigcap_{n} K_{n}$.

(c) A metric space for which no countably compact family can generate the topology: let $X$ be the dyadic numbers in $[0,1]$. Suppose, in fact, we had such a family $\mathscr{K}$. Let $x_{1}, x_{2}, \cdots$ be an enumeration of $X$. Then one could choose a sequence $K_{j}^{n}$ of neighborhoods of $x_{j}, K_{j}^{n} \in \mathscr{K}$, and with the length of $K_{j}^{n}$ less than $1 / n+j$. Let $U_{j}^{n}$ be the interior of $\overline{K_{j}^{n}}$. Then $x_{j} \in U_{j}^{n}$. Consider now the set $\bigcap_{n} \cup_{j} U_{j}^{n}$. This is a $G_{\delta}$ in the reals, and contains all the dyadics. Then it must contain some nondyadics, since the dyadics are not a $G_{\delta}$. On the other hand, if $\xi$ is a nondyadic in $\bigcap_{n} \bigcup_{j} U_{j}^{n}$, then $\xi$ is in some $\bigcap_{n} U_{j_{n}}^{n}$. Thus $\left\{K_{j_{n}}^{n} \mid n \in I\right\}$ has the F.I.P. But $\bigcap_{n} \overline{K_{j_{n}}^{n}}=\{\xi\}$, since the lengths of the $K_{j_{n}}^{n}$ go to zero as $n \rightarrow \infty$. Thus $\bigcap_{n} K_{j_{n}}^{n}=\bigcap_{n}\left(\overline{K_{j_{n}}^{n}} \cap X\right)=\phi$.

The question remains open whether, for example, every complete separable metric space has a countably compact family which generates its topology.

3. Measurability of various classes of functions. Throughout this section, let $X$ be a separable metric space; $\mathscr{X}$ the Borel sets. Let $\mathscr{K}$ be a collection of sets in $\mathscr{P}$ such that 
(a) $\mathscr{K}$ is a countably compact family,

(b) $\mathscr{K}$ generates the topology of $X$.

Let $T$ be a compact metric space, and consider $\mathscr{D}_{\mathscr{K}}, Q_{\mathscr{K}}$, as defined in $\S 1$. For brevity, we write simply $\mathscr{D}, Q$. We remark that the results of this section extend immediately to the case where $T$ is locally compact metrizable, and separable, since the classes of functions discussed are defined by their local properties in $T$.

Let $\mathscr{K}_{0}$ be a countable subset of $\mathscr{K}$ which still contains a complete system of neighborhoods at each point. Also, let $K_{\varepsilon, n}$ be an enumeration of the sets of $\mathscr{K}_{0}$ of diameter $\leqq \varepsilon$. Let $\Lambda(\varepsilon, S)=\bigcap_{s \in S}\{\omega \mid$ E some open neighborhood $U$ of $s$ and some $n$ such that $\omega$ sends $U$ into $\left.K_{\varepsilon, n}\right\}$. Finally, let $\Phi(\varepsilon, S)=\{\omega \mid \exists$ some open $U \supset S$ and $n$ such that $\omega$ sends $U$ into $\left.K_{\varepsilon, n}\right\}$.

Lemma 3.1. $\Lambda(\varepsilon, S)$ and $\Phi(\varepsilon, S)$ are in $\mathscr{D}$ for any closed set $S$ and any $\varepsilon>0$.

Proof. Let $\mathscr{U}$ be a countable base for the open sets of $T$. Let $\mathscr{U}_{1}, \mathscr{U}_{2}, \cdots$ be an enumeration of the finite coverings of $S$ by sets in U. Then $\Lambda(\varepsilon, S)=\bigcup_{n} \bigcup_{m} \bigcap_{U \in \mathscr{U}_{n}} \Delta\left(U, K_{\varepsilon, m}\right)$, and

$$
\Phi(\varepsilon, S)=\bigcup_{m} \bigcup_{n} \Delta\left(\bigcap_{\sigma \in \mathscr{U}_{n}} U, K_{\varepsilon, m}\right) .
$$

THEOREM 3.1. The set of all functions which are continuous at all points of the closed set $S \subset T$ is in $\mathscr{D}$.

Proof. This set is precisely $\bigcap_{m} \Lambda(1 / m, S)$.

THEOREM 3.2. For any regular measure $\nu$ on $T$, the set of $\nu$ almost everywhere continuous functions is in $\mathscr{D}$.

Proof. Let $V_{n, m}, n, m \in I$, be an enumeration of those finite unions of sets $\mathscr{U}$ such that $\nu\left(V_{n, m}\right)<1 / n$. A function $\omega$ is $\nu$-almost everywhere continuous if and only if for arbitrary small $\varepsilon>0$ there is a closed set $S$ whose complement has arbitrarily small measure, such that $\omega \in L(\varepsilon, S)$. But $\omega \in \Lambda(\varepsilon, S) \Rightarrow \omega \in L(\varepsilon, \bar{U})$ for some open set $U \supset S$. New, $S^{\perp}$ is a union of sets in $\mathscr{U}$. Since $S^{\perp} \supset U^{\perp}$, and $U^{\perp}$ is compact, $U^{\perp}$ is covered by a finite union of sets of $\mathscr{C}$ which does not intersect $S$, and thus has $\nu$-measure no greater than that of $S$. Hence, the set of $\nu$-almost everywhere continuous functions is contained in $\bigcap_{j} \bigcap_{n} \bigcup_{m} \Lambda\left(1 / j, V_{n, m}\right)$. The converse inclusion is obvious.

THEOREM 3.3. The set of functions whose points of discontinuity form a first category set, is in $\mathscr{D}$. 
Proof. Let $O_{\varepsilon}(\omega)=\{s \mid$ for every open $U \ni s \quad \exists \quad r, t \in U$ with $\mathrm{d}(\omega(r), \omega(t))>\varepsilon\} . \quad O_{\varepsilon}(\omega)$ is a closed set, and increases as $\varepsilon$ decreases. Thus, the set $\bigcup_{\varepsilon-0} O_{\varepsilon}(\omega)$ is of first category if and only if each $O_{\varepsilon}(\omega)$ is nowhere dense. Let $D$ be a countable densa subsat of $T$, and let $D_{n, m}$ be an enumeration of the finite $1 / m$-dense subsets of $D$ (i.e. every point of $T$ is within $1 / m$ of some point of $D_{n, m}$, for every $\left.n, m\right)$. Then following Nelson in Theorem 3.3 of [8], $O_{\varepsilon}(\omega)$ is nowhere dense if and only if, for every $m \in I, O_{\varepsilon}(\omega) \subset$ some $D_{n, m}^{\perp}$. Thus, $\omega$ has a first category set of discontinuities if and only if

$$
\omega \in \bigcap_{j} \bigcap_{m} \bigcup_{n} \ell\left(\frac{\perp}{\jmath}, D_{n, m}\right) .
$$

THEOREM 3.4. Let $T$ be a compact interval. Then the set of all $\omega$ with discontinuities of the first kind only, is in $\mathscr{D}$.

Proof. If $\omega$ has only discontinuities of the first kind, then for any $\varepsilon>0$ one can choose, for each $t \in T$, an open interval $R_{t}$ such that there are some fixed integers $n_{+}$and $n_{-}$for which $\omega(s) \in K_{\varepsilon, n_{+}}$for all $s$ in $\left(R_{t}-\{t\}\right)_{+} \cap T$ and $\omega(s) \in K_{\varepsilon, n_{-}}$for all $s$ in $\left(R_{t}-\{t\}\right)_{-} \cap T$. (Note :

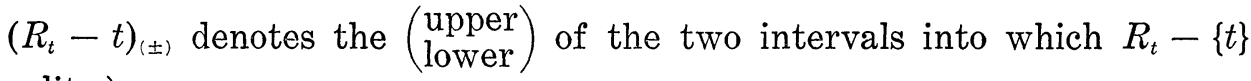
splits.)

Let $S_{t}$ be a rational open interval with $t \in S_{t} \subset \bar{S}_{t} \subset R_{t}$, and, for given $\delta>0$, let $U_{t}$ be another rational interval, of length $<\delta$, with $t \in U_{t} \cup S_{t}$. Then $\omega \in \Phi\left(\varepsilon,\left(\bar{S}_{t}-U_{t}\right)_{+} \cap T\right)$, and $\omega \in \Phi\left(\varepsilon,\left(\bar{S}_{t}-U_{t}\right) \cap T\right)$. Since $T$ can be covered by finitely many of the $S_{t}$, we finally get the following: let $\mathscr{S}_{1}, \mathscr{S}_{2}, \cdots$ be an enumeration of the finite coverings of $T$ by rational open intervals. For any rational open interval $S$, let $\mathscr{Q}_{k}(S)$ be the set of all open rational subintervals of $S$ having length $<1 / k$. Then if $\omega$ has only discontinuities of the first kind, we have $\omega \in \bigcap_{n} \bigcup_{m} \bigcap_{k} \bigcap_{s \in \mathscr{S}_{m}} \bigcup_{U \in \mathscr{U}_{k}(S)}\left\{\Phi\left(1 / m,(\bar{S}-U)_{+} \cap T\right) \cap \Phi\left(1 / n,(\bar{S}-U)_{-} \cap T\right)\right\}$. And conversely, if $\omega$ has a discontinuity of the second kind at $t_{0}$, then there is some integer $n$ such that no matter what open rational interval $S$ one chooses about $t_{0}, \omega$ will oscillate by more that $1 / n$ either in $(\bar{S}-U)_{+} \cap T$ or $(\bar{S}-U)_{-} \cap T$, provided $U$ is a sufficiently short interval. Thus, the inclusion is an equality.

Theorem 3.5. The set $\Theta$ of pairs $(\omega, t)$ in $\Omega \times T$ such that $\omega$ is discontinuous at $t$, is in $\mathscr{D} \times \mathscr{V}_{T}\left(\mathscr{T}_{T}\right.$ being the Borel sets in $\left.T\right)$. The function $(\omega, t) \rightarrow \omega(t) \mathscr{C} \times \mathscr{R}_{T} \mid \Theta^{\perp}-$ measurable, and a fortiori $\mathscr{D} \times \mathscr{T}_{T^{-}}$ measurable.

(Note: for a $\sigma$-algebra $\alpha$ on a set $Z$, and a set $Z_{0} \subset Z$, we denote by $\mathscr{A} \mid Z_{0}$ the $\sigma$-algebra $\left\{A \cap Z_{0} \mid A \in \mathscr{A}\right\}$. In case $Z_{0} \in \mathscr{A}$, we get

$$
\mathscr{A} \mid Z_{0}=\left\{A \in \mathscr{A} \mid A \subset Z_{0}\right\} \text {.) }
$$


Proof of Theorem 3.5. $\mathscr{U}$ is again a countable basis for the open sets of $T$. Then we have $\Theta^{\perp}=\bigcap_{n} \bigcup_{v \in \mathscr{U} \bigcup_{m}}\left[\Delta\left(U, K_{1 / n, m}\right) \times U\right]$. As for measurability of the function $(\omega, t) \rightarrow \omega(t)$ : let $T_{0}$ be a countable dense subset of $T$. Let $\mathscr{Y}_{k}$ be a finite covering of $T$ by sets of diameter $<1 / k$. Let $\left\{g_{k, V} \mid V \in \mathscr{Y}_{k}\right\}$ be a partititon of unity for $\mathscr{y}_{k}$. Let $f$ be a continuous function on $X$. Let $\widetilde{f}_{k}(\omega, t)=\sum_{\nabla \in \mathscr{U}_{k}} g_{k, V}(t) \sup _{s \in T_{0} \cap V} f(\omega(s))$. Then $\tilde{f}_{k}$ is $\mathscr{C} \times \mathscr{P}_{T}$-measurable, and, for fixed $\omega, f_{k}(t, \omega)$ is continuous in $t$. Furthermore, at all points $(\omega, t)$ in $\Theta^{\perp}$, we have $\tilde{f}_{k}(\omega, t) \rightarrow f(\omega(t))$. Thus, $f(\omega(t))$ is $\mathscr{C} \times \mathscr{B}_{T} \mid \Theta^{\perp}$-measurable for each continuous $f$. Now : for any closed set $K$ in $X$ there is a continuous function $f_{K}$ which is 1 only on that set. Then $\{(\omega, t) \mid \omega(t) \in K\} \cap \Theta^{\perp}=\left\{(\omega, t) \mid f_{K}(\omega(t))=1\right\} \cap \Theta^{\perp}$, which is in $\mathscr{C} \times \mathscr{B}_{T} \mid \Theta^{\perp}$. This completes the proof.

The generalization of Theorem 4.1 of [8] now goes through exactly as done there, by applying Fubini's theorem. Namely, if $\nu$ is a regular measure on $T$, then $\{\omega \mid \omega$ continuous at $t\}$ has $Q$-measure 1 for $\nu$-almost every $t \Longleftrightarrow\{t \mid \omega$ continuous at $t\}$ has $\nu$-measure 1 for $Q$-almost every $t \Longleftrightarrow \Theta$ has $Q \times \nu$-measure 0 . Similarly, Theorem 4.2 of [8] generalizes to the present context: if $\{\omega \mid \omega$ continuous at $t\}$ has $Q$-measure 0 for each $t \in T$, then $\{\omega \mid$ the discontinuities of $\omega$ form a cat $I$ set in $T\}$ has $Q$-measure 1 . The proof is gotten in the same way, but substituting $\widetilde{f}$ of Theorem 3.5 above for Nelson's $f^{+}$. The details will be omitted.

\section{REFERENCES}

1. J. L. Doob, Stochastic processes, Wiley, N. Y. (1943).

2. P. R. Halmos, Measure theory, van Nostrand, N. Y. (1950).

3. S. Kakutani, Notes on infinite product measure spaces, II, Proc. Imp. Acad. Tokyo, vol 19 (1943), pp. 184-188.

4. A. Khintchine, Asymptotische gesetze der Wahrscheinlichkeitsrechnung, Ergebnisse der Mathematik, vol. 2, no. 4.

5. A. Kolmogoroff Grundbegriffe der Wahrscheinlichkeitsrechnung, Ergebnisse der Mathematik, vol. 2 , no. 3 .

6. A. Mayer, Comptes Rendus, 248 (1959), 3106.

7. A. Mayer, Comptes Rendus, 249 (1959), 2475.

8. E. Nelson, Regular probability measures on function space, Annals of Math., 69 (1959), 630-643.

9. J. L. Kelley, General Topology, Van Nostrand, 1955. 


\section{PACIFIC JOURNAL OF MATHEMATICS}

\section{EDITORS}

Ralph S. Phillips

Stanford University

Stanford, California

M. G. Arsove

University of Washington

Seattle 5, Washington
A. L. Whiteman

University of Southern Californla

Los Angeles 7, California

Lowell J. Paige

University of California

Los Angeles 24, California

\section{ASSOCIATE EDITORS}

E. F. BECKENBACH

D. DERRY

H. L. ROYDEN

E. G. STRAUS

T. M. CHERRY

M. OHTSUKA

E. SPANIER

F. WOLF

\section{SUPPORTING INSTITUTIONS}

UNIVERSITY OF BRITISH COLUMBIA

STANFORD UNIVERSITY

CALIFORNIA INSTITUTE OF TECHNOLOGY

UNIVERSITY OF CALIFORNIA

MONTANA STATE UNIVERSITY

UNIVERSITY OF TOKYO

UNIVERSITY OF UTAH

UNIVERSITY OF NEVADA

NEW MEXICO STATE UNIVERSITY

OREGON STATE UNIVERSITY

UNIVERSITY OF OREGON

OSAKA UNIVERSITY

WASHINGTON STATE UNIVERSITY

UNIVERSITY OF WASHINGTON

UNIVERSITY OF SOUTHERN CALIFORNIA

AMERICAN MATHEMATICAL SOCIETY CALIFORNIA RESEARCH CORPORATION SPACE TECHNOLOGY LABORATORIES NAVAL ORDNANCE TEST STATION 


\section{Pacific Journal of Mathematics}

\section{Vol. 12, No. $1 \quad$ January, 1962}

Jonathan L. Alperin, Groups with finitely many automorphisms $\ldots \ldots \ldots \ldots \ldots \ldots \ldots \ldots$

Martin Arthur Arkowitz, The generalized Whitehead product ................ 7

John D. Baum, Instability and asymptoticity in toplogical dynamics . . . . . . . . . . 25

William Aaron Beyer, Hausdorff dimension of level sets of some Rademacher series .... $\quad 35$

Frank Herbert Brownell, III, A note on Cook's wave-matrix theorem . . . . . . . . . . . . . 47

Gulbank D. Chakerian, An inequality for closed space curves ................. 53

Inge Futtrup Christensen, Some further extensions of a theorem of Marcinkiewicz ....... 59

Charles Vernon Coffman, Linear differential equations on cones in Banach spaces . . . . . 69

Eckford Cohen, Arithmetical notes. III. Certain equally distributed sets of integers . . . . . 77

John Irving Derr and Angus E. Taylor, Operators of meromorphic type with multiple poles

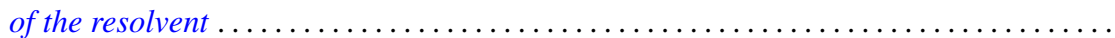

Jacob Feldman, On measurability of stochastic processes in products space .............

Robert S. Freeman, Closed extensions of the Laplace operator determined by a general class of boundary conditions, for unbounded regions ......................

Robert E. Fullerton, Geometric structure of absolute basis systems in a linear topological

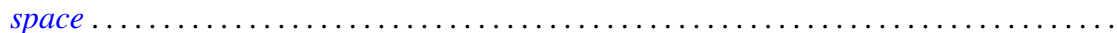

Dieter Gaier, On conformal mapping of nearly circular regions

Andrew Mattei Gleason and Hassler Whitney, The extension of linear functionals defined

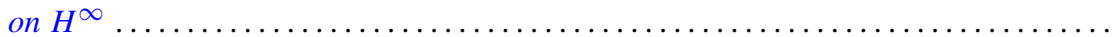

Seymour Goldberg, Closed linear operators and associated continuous linear

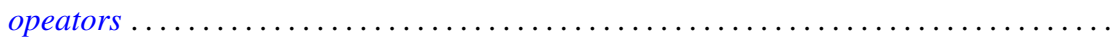

Basil Gordon, Aviezri Siegmund Fraenkel and Ernst Gabor Straus, On the determination of sets by the sets of sums of a certain order

Branko Grünbaum, The dimension of intersections of convex sets. .

Paul Daniel Hill, On the number of pure subgroups

Robert Peter Holten, Generalized Goursat problem . .

Alfred Horn, Eigenvalues of sums of Hermitian matrices ...........

Henry C. Howard, Oscillation and nonoscillation criteria for

$$
y^{\prime \prime}(x)+f(y(x)) p(x)=0
$$

Taqdir Husain, $S$-spaces and the open mapping theorem ...

Richard Eugene Isaac, Markov processes and unique stationary probability measures ...

John Rolfe Isbell, Supercomplete spaces ....................

John Rolfe Isbell, On finite-dimensional uniform spaces. II .........

N. Jacobson, A note on automorphisms of Lie algebras ..............

Antoni A. Kosinski, A theorem on families of acyclic sets and its applications

Marvin David Marcus and H. Minc, The invariance of symmetric functions of singular values...

Ralph David McWilliams, A note on weak sequential convergence.

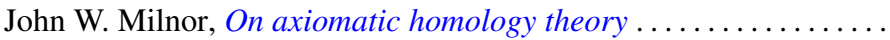

Victor Julius Mizel and Malempati Madhusudana Rao, Nonsymmetric projections in

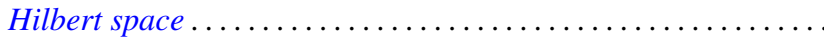

Calvin Cooper Moore, On the Frobenius reciprocity theorem for locally compact

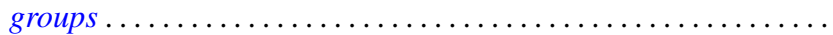

Donald J. Newman, The Gibbs phenomenon for Hausdorff means . 